\title{
How closely-knit is the martian atmosphere system?
}

A community white paper submitted to

the Committee for the Decadal Survey on Planetary Science and Astrobiology 2023 - 2032

Primary author:

Majd Mayyasi (Boston University, USA, majdm@bu.edu)

Co-authors:

Beatriz Sánchez-Cano (University of Leicester, UK, bscmdr1 @leicester.ac.uk)

Kerstin Peter (Universität zu Köln, Germany, peterk@uni-koeln.de)

Mehdi Benna (NASA Goddard Space Flight Center, USA, mehdi.benna-1@ nasa.gov)

Robert Lillis (University of California Berkeley, USA, rlillis@ @ssl.berkeley.edu)

Robin Ramstad (University of Colorado Boulder, USA, Robin.Ramstad@ lasp.colorado.edu)

Xiaohua Fang (University of Colorado Boulder, USA, Xiaohua.Fang@ lasp.colorado.edu)

Christopher Fowler (University of California Berkeley, USA, cmfowler@berkeley.edu)

Guillaume Gronoff (SSA/NASA LaRC, USA, guillaume.p.gronoff@nasa.gov)

Mika Holmberg (European Space Agency, Netherlands, Mika.Holmberg@esa.int)

Stephen Bougher (University of Michigan, USA, bougher@umich.edu)

Mark Lester (University of Leicester, UK, mle@leicester.ac.uk)

Bruce Jakosky (University of Colorado Boulder, USA, bruce.jakosky @lasp.colorado.edu)

Janet Luhmann University of California Berkeley, USA, jgluhman@ ssl.berkeley.edu)

John Clarke (Boston University, USA, jclarke@bu.edu)

David Brain (University of Colorado Boulder, USA, David.Brain@lasp.colorado.edu)

David L. Mitchell (University of California Berkeley, USA, mitchell@ssl.berkeley.edu)

Michael Chaffin (University of Colorado Boulder, USA, michael.s.chaffin@gmail.com)

Supporters:

Alexander Shane
Anders Eriksson
Andrew Kopf
Chaufray Jean-Yves
Christian Mazelle
Christina O. Lee
Chuanfei Dong
Clara Narvaez
David Andrews
Dolon Bhattacharyya
Ed Thiemann
Edik Dubinin
Gangkai Poh
Gina A DiBraccio
Guillaume Gronoff
Gwen Hanley

J. Scott Evans
Janet Luhmann
Jim Wild
Karim Meziane
Katerina Stergiopoulou
Kim Konstantin
Leonardo Regoli
Manuel Grande
Marek Slipski
Markus Fraenz
Martin Pätzold
Matteo Crismani
Matthew Fillingim
Mike Liemohn
Nicolas Andre
Norberto Romanelli

Oleg Vaisberg

Paul Mahaffy

Scott Thaller

Sergey Shuvalov

Shane W. Stone

Shotaro Sakai

Silvia Tellmann

Stas Barabash

Suranga Ruhunusiri

Thomas Cravens

Tracy Esman

Yaxue Dong

Yingjuan Ma

Yuni Lee

Zachary Girazian

Zhaojin Rong 


\section{Executive Summary}

The Mars system encompasses interactional processes at the surface and regolith at one boundary and solar inputs at its other boundary where atmospheric particles escape. The spatial boundaries and regions within need to be investigated simultaneously to better understand the strong coupling that the atmosphere of Mars has within its different altitude regions, within its different atmospheric populations, and with external solar inputs. These investigations are most fruitful when examined at temporal timescales that cover short-duration processes such as solar weather events, as well as longer duration processes that track seasonal and solar variations.

\section{Recommendations}

The NASA's Decadal Survey recommendations for future Mars missions are as follows:

1) Continued support for MAVEN during its extended mission due to unique capabilities of the instrument suite and upper atmospheric coverage that address solar-upper atmospheric coupling, and multi-species coupling in the upper atmosphere of Mars with broad temporal coverage.

2) Promotion of multi-point mission concepts to Mars, such as MOSAIC, with a high yield of extensive spatial coverage from surface to space to address the coupling within regions near the regolith and how these processes transport to higher altitudes.

3) Consideration of instrumentation development that will tackle broad gaps in present understanding of the planetary system. A multi-channel mass spectrometer, highsensitivity UV echelle detector, and retarding potential analyzer are suggested to tackle the gaps in determining atmospheric propagation and escape as well as the heating budget at Mars.

4) Prioritizing missions to Mars over the next decade that emphasize cohesive and interdisciplinary science goals to enable better understanding of the planet as a system. 


\section{Brief Overview}

Mars is complicated.

The importance of understanding the present climate of Mars and its past evolution as well as implications for past biological life, and prospects for future human habitability are key topics highlighted in the most recent NASA decadal survey. Abundant evidence of fluvial activity indicates the planet was once warm and at least episodically wet, with conditions conducive to harboring life 1 . Today, Mars is cold and dry with a surface pressure less than $1 \%$ that of Earth. What happened to most of the planet's atmosphere, the liquid water, and how quickly the martian environment changed from habitable to harsh, are still open questions that have interested global communities.

While several past Mars landers have examined the surface properties for insights into biotic signatures, NASA orbiting missions have begun to unveil some of the processes that drive atmospheric loss. Necessary elements toward achieving this goal lie in understanding the seasonal cycles within the martian atmosphere, how these cycles affect atmospheric species, and how these cycles vary from surface to space.

The upper atmosphere of Mars is the reservoir for escape of matter from the planet. Understanding the evolution and escape of the martian atmosphere requires an understanding of the atmosphere below the escape region, and how that atmosphere connects to the surface. The processes that replenish, drive and then remove species from the topmost region of Mars are not yet well understood. Variability of atmospheric species near the surface is not reflected in the upper regions where atoms from these species escape. To understand this propagation and alteration of atmospheric properties requires simultaneous multi-altitude measurements at various timescales.

Water loss from Mars demonstrates this challenge. The properties of water reservoirs in the subsurface, in polar caps, and in the near-surface atmosphere vary significantly with martian season2,3,4,5,6,7,8,9. Atmospheric circulation uplifts water into higher altitudes that are affected by dust, ice and vapor clouds. Solar input and atmospheric chemistry contribute to ionizing and/or dissociating water molecules into constituent atoms, thereby altering the physical drivers that affect water dynamics and further propagation to higher altitudes10,11. Gravity waves12,13,14,

\footnotetext{
${ }^{1}$ Carr, 2012, The Royal Society 370 (1966), doi: 10.1098/rsta.2011.0500

2 Farmer et al., 1977, Journal of Geophysical Research, 82, 28.

${ }^{3}$ Owen et al., 1988, 240, $1767-1770$.

${ }^{4}$ Bertaux and Montmessin, 2001, Journal of Geophysical Research, 106, 32, 879-33994 E12.

${ }^{5}$ Maltagliati et al., 2007, Icarus, 597 - 602, doi:10.1016/j.icarus.2007.09.027.

${ }^{6}$ Smith et al., 2009, Journal of Geophysical Research, 114, E00D03.

${ }^{7}$ Novak et al., 2011, Planet. Space Sci., 59, $163-168$.

${ }^{8}$ Villanueva et al., 2015, Science, 348(6231), 218-221, doi:10.1126/science.aaa3630.

${ }^{9}$ Aoiki et al., 2015, Icarus, 7-22, doi:10.1016/j.icarus.2015.06.21.

${ }^{10}$ Fedorova et al., 2020, Science, doi:10.1126/science.aay9522.

11 Encrenaz et al., 2018, Astro. \& Astrophys., 612, A112, doi:10.1051/0004-6361/201732367.

12 England et al., 2017, Journal of Geophysical Research: Space Physics 122 (2), 2310-2335.

13 Terada et al., 2017, Journal of Geophysical Research: Space Physics 122 (2), 2374-2397.

14 Yigit et al., 2015, Geophys. Res. Lett. 42 (21), 8993-9000.
} 
regional and global dust storms 1,2,3, and space weather events4,5,6 are a few of the drivers that have been recently observed to alter the properties of the atmosphere at Mars at various altitudes7,8,9,10,11.

The upper atmosphere is also a region populated by charged and neutral species. The charged gas within the upper atmosphere of Mars, a region called the ionosphere, was previously thought to be a mostly independent fluid that only originated from the ambient neutral atmosphere. Investigations facilitated by the NASA MGS 12 and MAVEN 13,14 missions have shown that strong coupling exists between the neutral and ionized components of the atmospheres, although they are each governed by different physical processes15. How these two fluids, that are comprised of multiple species, evolve is a multi-disciplinary question that requires a surface-to-space assessment of atmospheric properties and their variability.

\section{Atmospheric Coupling: Existing Enigmas}

Figure 1 demonstrates the numerous processes presently known to affect different atmospheric regions at Mars. Several unexplained phenomena persist.

Case for Water: Water $\left(\mathrm{H}_{2} \mathrm{O}\right)$ is best measured in conjunction with its isotopologue, HDO, to facilitate tracing water content to primordial times at Mars. At lower altitudes, water and HDO vary with similar near-surface circulation patterns. Above $\sim 10 \mathrm{~km}$, these molecules interact differently with aerosols and clouds. Circulation processes lift up these molecules to higher altitudes where solar photons dissociate them into their constituent atoms. Despite a mass difference as small as $1 \mathrm{amu}, \mathrm{H}$ diffuses to much higher altitudes than $\mathrm{D}$ and therefore escapes the planet much more readily. As with their parent molecules at lower altitudes, D and $\mathrm{H}$ abundances in the upper atmosphere are used as a metric to estimate water loss. Multiple missions to Mars have measured $\mathrm{D}$ and $\mathrm{H}$ at the surface, lower atmosphere, and upper atmosphere, yet these measurements do not reconcile because we do not yet understand the multitude of processes that affect propagation of water, as well other atmospheric species, up from the surface. Measurements of water and its isotopes at various altitudes would be needed to understand the extent and coupling of water to the atmospheric cycle.

\footnotetext{
${ }^{1}$ Kliore et al., 1973, J. Geophys. Res., 78(20), 4331-4351, doi:10.1029/JB078i020p04331.

2 Smith et al., 2002, Icarus, 157(1), 259-263, doi:10.1006/icar.2001.6797.

${ }^{3}$ Fang et al., 2020, J. Geophys. Res., 125, doi:10.1029/2019JA026838.

4 Jakosky et al., 2015a, Science 350 (6261), doi: 10.1126/science.aad0210.

${ }^{5}$ Lee et al., 2018, Geophysical Research Letters 45 (17), 8871-8885.

${ }^{6}$ Mayyasi et al., 2018, Geophysical Research Letters 45 (17), 8844-8852.

${ }^{7}$ Heavens et al., 2018, Nature Astronomy 2, 126-132, doi: 10.1038/s41550-017-0353-4.

${ }^{8}$ Vandaele et al., 2019, Nature 568, 521-525.

${ }^{9}$ Kass et al., 2019, Geophysical Research Letters, doi: 10.1029/2019GL083931.

${ }^{10}$ Kleinbohl et al., 2020, Geophysical Research Letters 125 (1), doi: 10.1029/2019JE006115.

11 Girazian et al., 2020, Journal of Geophysical Research: Planets 125 (5), e2019JE006092.

12 Albee et al., 1998, Science 279 (5357), 1671-1672, doi: 10.1126/science.279.5357.1671.

13 Jakosky et al., 2015b, Geophysical Research Letters 42 (21), 8791-8802, doi: 10.1002/2015GL065271.

14 Jakosky et al., 2015c, Space Science Reviews 195 (1), 3-48, doi: 10.1007/s11214-015-0139-x.

15 Mayyasi et al., 2019, Journal of Geophysical Research: Space Physics, 124, doi:10.1029/2019JA026481.
} 
Case for Energy: Solar extreme ultraviolet and X-ray photons impinging on the neutral atmosphere of Mars ionize species to generate ions and electrons during the day that mostly recombine during the night $1,2,3$. These solar photons also instigate heating of the upper atmospheric plasma and drive the dynamical processes that are inter-related between charged particle populations. Understanding atmospheric heating requires understanding the energy inputs (solar photons4, solar wind particles, wave-particle interactions 5,6) at the top of the atmosphere, as well as understanding the strong coupling between electrons, ions, and neutral species at lower altitudes where energy is absorbed. To date, theoretical assessments of atmospheric heating using solar photons alone have not reproduced observed temperatures. Further investigations are limited due to the dearth in ion temperature measurements, and due to the lack of availability of concurrent observations of ambient electric and magnetic field as well as solar wind parameters. The energy budget at Mars remains a mystery.

Case for Gases: Populations of charged particles are governed by different dynamics and energization than their ambient neutrals. The MAVEN mission made the first continuous simultaneous observations of both ionized and neutral species in the upper atmosphere of Mars. Investigations have shown that these two populations are tightly coupled, and that they vary considerably over observational timescales 7,8 . The driver of such tightly-coupled variability is still poorly understood. Lower-altitude generated atmospheric tides and gravity waves are candidate drivers of this variability, but supporting measurements are not sufficient. Observations at lower altitudes would be required to determine what how coupling with the upper atmosphere occurs.

The variability of the martian atmosphere includes many unknowns. Fundamental to understanding atmospheric drivers and the extent of their coupling is to make measurements that cover a range of altitudes simultaneously. Processes that connect variability in lower, middle, and upper atmospheres and multiple gases within, can be understood using spatial multipoint observations on timescales relevant to known drivers such as: solar flares (minutes), energetic particle events and coronal mass ejections (hours), dust storms (weeks), seasons (months), and solar cycles (decades). For such measurements to be comprehensive, they need to span various topographical locations and local times.

\section{Open Science Questions}

Mars landers cover examination of a relatively small geographical region at the surface, and orbiters cover local-time-restricted and/or altitude restricted regions of the middle-to-upper atmosphere. These restrictions have limited understanding of Mars into relatively numerous yet narrow bands of applicability. A comprehensive understanding of how Mars behaves and evolves

\footnotetext{
${ }^{1}$ Fox et al., 1996, Advances in Space Research 17 (11), 203-218, doi: 10.1016/0273-1177(95)00751-Y.

2 Benna et al., 2015, Geophysical Research Letters 42 (21), 8958-8965.

${ }^{3}$ Girazian et al., 2017, Journal of Geophysical Research: Space Physics, 122, doi:10.1002/2016JA023508.

${ }^{4}$ Fang et al., 2019, Geophysical Research Letters, 46, 9334-9343, doi:10.1029/2019GL084515.

${ }^{5}$ Fowler et al., 2018, Journal of Geophysical Research: Space Physics, 123, doi:10.1029/2018JA025208.

${ }^{6}$ Fowler et al., 2020, Geophysical Research Letters, doi:10.1029/2019GL086408.

${ }^{7}$ Bougher et al., 2004, Journal of Geophysical Research: Planets, 109(E3), doi:10.1029/2003JE002154.

${ }^{8}$ Cahoy et al., 2006, Journal of Geophysical Research: Planets, 111(E5), doi:10.1029/2005JE002634.
} 
in the present epoch is an attainable goal with present resources, instrument heritage, and innovation. A few open science questions are emphasized toward this goal:

- What traps water closer to the surface in some regions and not others? And, how does water traverse the cloud layers to reach higher altitudes?

- How do the atmospheric effects of dust storms propagate from near surface into the upper atmosphere? And how does that affect atmospheric structure and loss to space?

- Are local and regional dust storms competitive drivers of global circulation patterns and atmospheric loss?

- What are the major processes that generate atmospheric variability at lower altitudes?

- Does the solar wind interaction comprehensively account for the missing heating components in the atmosphere of Mars? Do particles alone account for this heating discrepancy or do electric and magnetic fields impart a significant amount as well?

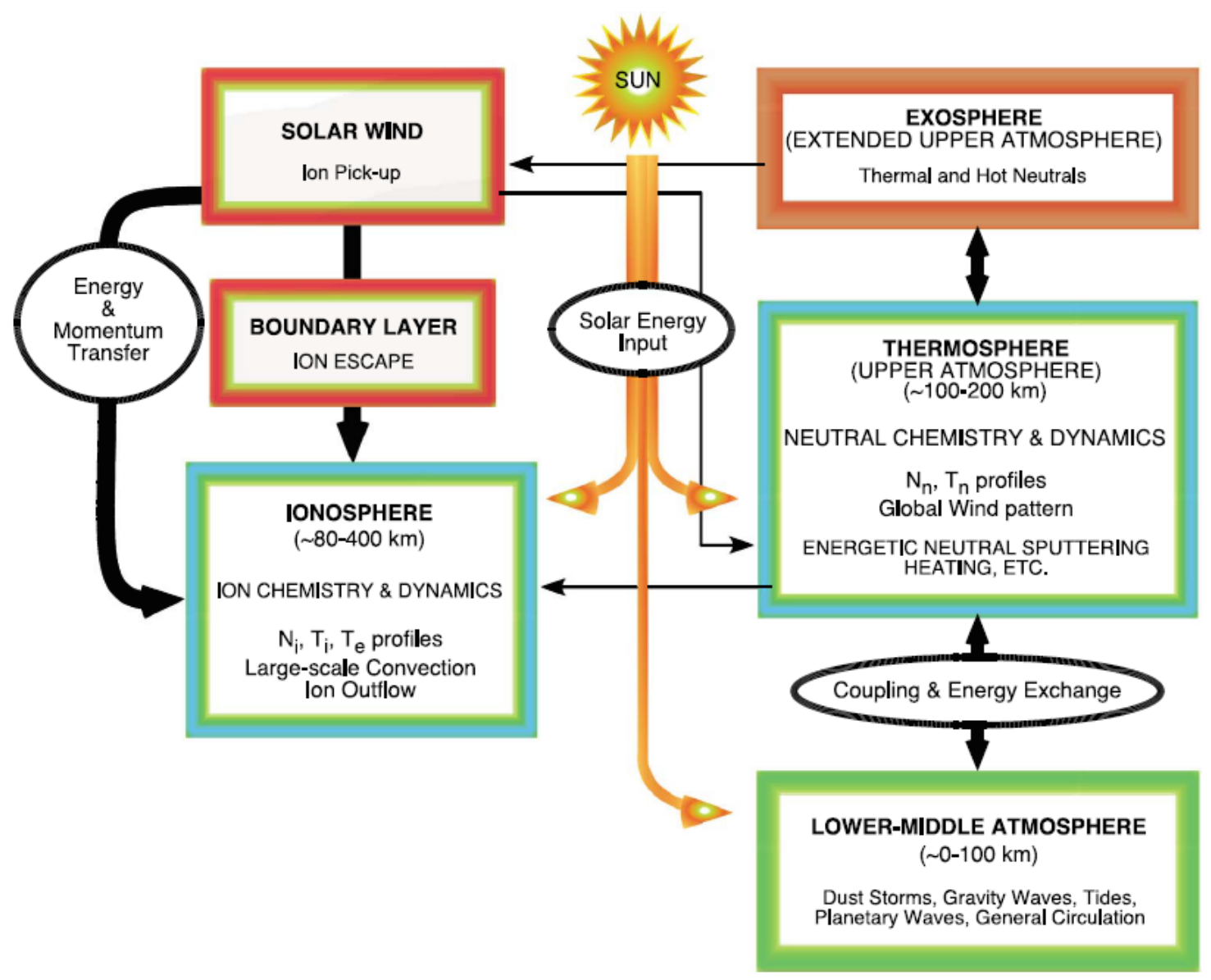

Figure 1: Cartoon of Mars atmospheric regions and processes. Illustrates the coupling of the thermosphere and ionosphere with atmospheric regions above and below1.

${ }^{1}$ Bougher at al., 2015, JGR Planets, 120, 311-342, doi:10.1002/2014JE004715. 


\section{Filling in the Gaps}

The Viking Landers included the first mass spectrometers at Mars, and provided two-point measurements, during descent, of the in situ composition of major neutral and ion species. Since then, the MAVEN NGIMS instrument has provided compositional information from orbit, for a wider range and higher sensitivity of species (2-150 amu).

$\rightarrow$ GAP1: key species such as $\mathrm{H}, \mathrm{H}_{2}$ and $\mathrm{D}$ are at or below the lower limits of the spectrometer channel where density retrievals are most challenged, if not impossible to obtain with the current configuration. Such measurements can provide high impact in situ observations of H and D densities directly and would contribute significantly to identifying the $\mathrm{D} / \mathrm{H}$ ratio at spacecraft orbiting altitudes.

Species with faint emissions in the UV include D Lyman- $\alpha(121.5 \mathrm{~nm})$ and O doublet at 135.6 $\mathrm{nm})$. At Mars, these faint emissions are optically thin and therefore much easier to interpret than optically thick emissions (such as H Lyman- $\alpha$ and O triplet at $130.4 \mathrm{~nm}$ ). The Hubble Space Telescope STIS instrument includes a high-sensitivity UV echelle spectrograph, capable of measuring faint emissions at Mars as low as 30 Rayleigh, as it orbits Earth. The MAVEN IUVS spectrometer echelle channel makes similar measurements from Mars orbit, yet is challenged by noise on the detector that significantly prohibits detections below $\sim 150$ Rayleigh.

$\rightarrow$ GAP2: MAVEN IUVS retrievals of D properties are limited to about half of the martian year (when the planet is around perihelion, and the $\mathrm{D}$ emission is brightest). This prohibits observation of the full seasonal variability of $\mathrm{D}$ and $\mathrm{H}$ from the upper atmosphere of Mars, where water components escape. It would be more feasible to fly a mission to Mars with a highly sensitive echelle spectrograph than to get routine observational time on HST.

At most thermosphere altitudes, charged species at Mars are highly collisional. Inter-particle dynamics and ambient heating/cooling are tightly coupled processes that depend on the temperatures of the particles. Theoretical investigations have shown that lighter ions can get up to $30 \%$ hotter than heavier ones1, affecting their interactions with other species, and making them more prone to escape.

$\rightarrow$ GAP3: ion temperatures at Mars have only ever been measured by the Viking Lander descent probes. Spatially and temporally broader measurements of ion temperatures of multiple martian ion species are needed to understand fundamental plasma dynamics that govern escape/retention of the charged gases at Mars.

Critical and novel measurements for consideration at Mars that are highly relevant to cohesively understanding the planetary system are recommended below. These instrument recommendations address the fundamental knowledge gaps, and address the open science questions:

1) Orbiter: a multi-channel mass spectrometer with the range and sensitivity to measure water molecular and atomic isotopes (neutral and ionized) at multiple altitudes. Such an instrument

${ }^{1}$ Matta et al., 2014, Icarus, 227, 78-88, doi:10.1016/j.icarus.2013.09.006 
would ideally be on an orbiter with a spacecraft periapsis-apoapsis range that adequately samples the thermosphere $(\sim 100-400 \mathrm{~km})$.

2) Orbiter: a high-sensitivity UV echelle spectrograph to measure low emission species such as deuterium in Lyman-alpha $(121.5 \mathrm{~nm})$, and the forbidden $O$ doublet line $(135.6 \mathrm{~nm})$, both of which are optically thin and ideal for scientific interpretation of the loss of atmospheric species at Mars.

3) Orbiter: a retarding potential analyzer capable of measuring the ion temperature at Mars from orbit. With heritage from the Viking Lander descent probes, ion temperature can be used with existing MAVEN electron temperature and solar wind energetic particle measurements to determine what heats up Mars to its observed state.

These instruments would complement orbiter components of a multi-point mission concept to resolve the science gaps. Such instrument concepts exist and would need to be customized for the appropriate science application in low-risk high-science yield ways.

\section{Existing and New Mission Concepts}

The NASA MAVEN mission has done much to disambiguate and characterize the atmosphere of Mars and how it interacts with solar drivers. Just as previous long-lived Mars orbiters, the scientific yield of a mission deployed to a dynamical system increases with the lifetime of its measurements. This white paper is in support of a continued scientific investigation of Mars with MAVEN both as a standalone mission covering temporal variability, as well as a synergistic mission to optimize concurrent spatial coverage with observations from TGO and potentially EMM. MAVEN's unique mass spectrometer capabilities will specifically be able to address coupling at an atmospheric composition level, at altitudes of operation that are narrowing with extended mission timeline.

The MOSAIC mission concept is ideal for unveiling the rich and dynamic coupling processes within the martin atmosphere, by making the required simultaneous measurements of atmospheric water, aerosols, winds, temperatures and ice properties. MOSAIC thus has the potential to separate the effects of dust and seasonal cycles on the variability of species at higher altitudes, and atmospheric escape rates. MOSAIC's swarm of spacecraft will address many fundamental questions of what triggers variability as observations are made simultaneously at multiple atmospheric regions.

\section{Conclusion}

Mars exploration is a target for multiple space agencies due to its proximity to Earth as well as to its conditions that may have supported early life. MEPAG, and the Mars Architecture Strategy Working Group (MASWG) highlight the importance of understanding Mars as a system for human exploration and habitation, specifically, an understanding of Mars's habitability and changing climate, its evolution as a standalone system, and its capacity as a model to infer exoplanetary atmospheric evolution 1.

${ }^{1}$ Gronoff et al., 2020, J. Geophys Res., doi:10.1029/2019JA027639 